\title{
The relative abundance of wheat Rubisco activase isoforms is post-transcriptionally regulated
}

\author{
Juan Alejandro Perdomo ${ }^{1}$ (i) $\cdot$ Peter Buchner ${ }^{2}$. Elizabete Carmo-Silva ${ }^{1}$
}

Received: 2 December 2020 / Accepted: 11 March 2021 / Published online: 1 April 2021

(c) The Author(s) 2021

\begin{abstract}
Diurnal rhythms and light availability affect transcription-translation feedback loops that regulate the synthesis of photosynthetic proteins. The $\mathrm{CO}_{2}$-fixing enzyme Rubisco is the most abundant protein in the leaves of major crop species and its activity depends on interaction with the molecular chaperone Rubisco activase (Rca). In Triticum aestivum L. (wheat), three Rca isoforms are present that differ in their regulatory properties. Here, we tested the hypothesis that the relative abundance of the redox-sensitive and redox-insensitive Rca isoforms could be differentially regulated throughout light-dark diel cycle in wheat. While TaRcal- $\beta$ expression was consistently negligible throughout the day, transcript levels of both TaRca2- $\beta$ and TaRca2- $\alpha$ were higher and increased at the start of the day, with peak levels occurring at the middle of the photoperiod. Abundance of TaRca- $\beta$ protein was maximal $1.5 \mathrm{~h}$ after the peak in TaRca2- $\beta$ expression, but the abundance of TaRca- $\alpha$ remained constant during the entire photoperiod. The redox-sensitive TaRca- $\alpha$ isoform was less abundant, representing $85 \%$ of the redox-insensitive TaRca- $\beta$ at the transcript level and 12.5\% at the protein level. Expression of Rubisco large and small subunit genes did not show a consistent pattern throughout the diel cycle, but the abundance of Rubisco decreased by up to $20 \%$ during the dark period in fully expanded wheat leaves. These results, combined with a lack of correlation between transcript and protein abundance for both Rca isoforms and Rubisco throughout the entire diel cycle, suggest that the abundance of these photosynthetic enzymes is post-transcriptionally regulated.
\end{abstract}

Keywords Rubisco $\cdot$ Rubisco activase $\cdot$ Gene expression $\cdot$ Protein abundance $\cdot$ Diel cycle

\section{Introduction}

Photosynthesis is one of the most important physiological processes in plants that begins with the absorption of light energy and leads to fixation of $\mathrm{CO}_{2}$ (Berry and Downton 1982). Photosynthesis is regulated by an internal timekeeping system, the circadian clock, which runs in a period of $24 \mathrm{~h}$ and regulates several molecular and physiological processes such as growth, enzyme activity and control of stomatal aperture (Harmer 2009). In this study, we set out to investigate whether the abundance of ribulose-1,5-bisphosphate carboxylase/oxygenase (Rubisco) and its molecular chaperone, Rubisco activase (Rca), change in concert with

Juan Alejandro Perdomo

alejandro.perdomo@lancaster.ac.uk

1 Lancaster Environment Centre, Lancaster University, Lancaster LA1 4YQ, UK

2 Plant Biology and Crop Science Department, Rothamsted Research, Harpenden AL5 2JQ, UK light availability throughout the diel cycle. Wheat (Triticum aestivum L.) is one of the most important crops worldwide, supplying more than $20 \%$ of the calories consumed by humanity (Ray et al. 2013). We have previously shown that wheat contains three Rca isoforms (Carmo-Silva et al. 2015) that differ in their regulatory properties (Perdomo et al. 2019). Here, we tested the hypothesis that the relative abundance of the three Rca isoforms in wheat leaves would change throughout the day, with a potential impact on Rubisco activity.

Rubisco consists of eight large subunits (LSU), encoded by a single gene $(r b c L)$ in the chloroplast, and eight small subunits (SSU), encoded by a multigene family $(R b c S)$, in the nuclear genome (Schmidt and Mishkind 1986; Roy 1989). Rubisco is the most abundant protein in plants and is responsible for net $\mathrm{CO}_{2}$ assimilation. However, Rubisco has been described as one of the most inefficient enzymes due to its very low catalytic turnover rate and the susceptibility to inhibition by unproductive binding of sugar-phosphate derivatives that lock the active sites in a closed conformation 
(Brooks and Portis 1988; Jordan and Chollet 1983; Portis 1995). Rubisco activase (Rca) is a catalytic chaperone of Rubisco belonging to the AAA+ protein family (Neuwald et al. 1999). Rca uses the energy from ATP hydrolysis to restore the catalytic competence of Rubisco by promoting the release of the inhibitory sugar-phosphates from Rubisco active sites (Portis 1995).

In many flowering plant species, Rca exists as two isoforms that are almost identical except for a 30-39 amino acid extension of the $\mathrm{C}$ terminus that is present only in the longer Rca- $\alpha$ isoform and differentiates it from the shorter Rca- $\beta$ isoform (Salvucci et al. 1987; Werneke et al. 1989). Two cysteine residues at the C-terminal extension confer redox-sensitivity to Rca- $\alpha$ (Zhang and Portis 1999; Zhang et al. 2001). The Rca- $\alpha$ and Rca- $\beta$ isoforms are the products of either alternative splicing or separate genes depending on the species. Three Rca isoforms, two $\beta$ and one $\alpha$, are encoded by two genes in wheat (Carmo-Silva et al. 2015). Expression of the TaRcal gene produces a Rca1- $\beta$ isoform, whereas alternative splicing of the TaRca2 gene produces either a Rca2- $\beta$ or a Rca2- $\alpha$ isoform. The gene expression and abundance of Rca isoforms varies considerably among species, with Rca- $\alpha$ sometimes present in similar amount, but generally much less abundant than Rca- $\beta$ (Salvucci et al. 1987, 2003; Yin et al. 2010).

Rca gene expression in higher plants is almost entirely restricted to green tissues, and is developmentally regulated by leaf age as well as regulated by light (Orozco and Ogren 1993; Watillon et al. 1993; Liu et al. 1996). The expression of Rca of numerous species including Arabidopsis, tomato, apple and rice is regulated by the circadian clock (Pilgrim and McClung 1993; Martino-Catt and Ort 1992; Watillon et al. 1993; To et al. 1999). In Arabidopsis and tomato, these circadian patterns are evident in the rate of net protein synthesis alongside with the accumulation of mRNA levels (Martino-Catt and Ort 1992; Pilgrim and McClung 1993). However, a physiological role for a circadian rhythm in Rca transcript accumulation is unclear and the level of Rca protein in mature tobacco leaves does not exhibit a similar oscillation (Klein and Salvucci 1995). It has been proposed that a physiological role in photosynthetic performance may exist in early stages of leaf development as Rca levels are low and Rca transcript abundance enhanced by circadian rhythm could impact protein abundance (Martino-Catt and Ort 1992; Pilgrim and McClung 1993).

Rubisco $r b c L$ and $R b c S$ mRNA amounts fluctuate during the diurnal cycle with different patterns in different species; however, only the $R b c S$ subunit follows a circadian rhythm (Pilgrim and McClung 1993; Recuenco-Muñoz et al. 2015). In rice, $r b c L$ and $R b c S$ amounts fluctuated during diurnal cycle with peak abundance levels during the light phase of the photoperiod (Wang and Wang 2011). In Arabidopsis plants grown in a light/dark photoperiod, $R b c S$
mRNA exhibits a diurnal pattern of expression, with peak abundance occurring soon after beginning of the light and minimum levels at the end of the light period (Pilgrim and McClung 1993). In contrast, the amount of $r b c L$ and $R b c S$ transcripts in Chlamydomonas reinhardtii were highest in the dark (Recuenco-Muñoz et al. 2015). Other studies with $C$. reinhardtii indicate that $r b c L$ mRNA levels are not directly correlated to the amount of functional Rubisco protein (Winder et al. 1992; Cohen et al. 2006). This lack of correlation between gene expression and protein abundance suggests that the latter is post-transcriptionally regulated, and that transcript levels are insufficient to predict functional protein levels (Vogel and Marcotte 2012; Liu et al. 2016).

Despite the characterisation of Rca isoforms in wheat (Carmo-Silva et al. 2015; Perdomo et al. 2019) and gene expression studies under abiotic stresses such as drought and temperature (Zhao et al. 2017; Scafaro et al. 2019a; Degen et al. 2021) little is known about diurnal regulation of their abundance. The three wheat Rca activate Rubisco at different rates and differ in their sensitivity to light stimuli, changes in the ADP/ATP ratio (Perdomo et al. 2019). These findings suggest that changes in the relative abundance of each isoform could affect the rate of $\mathrm{CO}_{2}$ fixation by Rubisco in wheat leaves. The aim of this study was to characterise the expression levels of Rubisco and the three Rca isoforms present in wheat during the $24 \mathrm{~h}$ diel cycle and investigate whether the diurnal fluctuations in gene expression are translated into protein abundance. Likewise, we wanted to identify the time period when the protein amounts are highest and constant during the day.

\section{Materials and methods}

\section{Plant material}

Plants of T. aestivum L. cultivar Cadenza were grown from seed in $1.5 \mathrm{~L}$ pots $(11 \times 11 \mathrm{~cm})$ containing Rothamsted description mix compost with $75 \%$ medium grade (L\&P) peat, $12 \%$ screened sterilised loam, 3\% medium grade vermiculite, $10 \%$ grit ( $5 \mathrm{~mm}$ screened, lime free). After 2 weeks, plants were thinned down to have 3 plants per pot, with a total of 90 plants (30 pots). Plants were grown in controlled environment cabinets (Fitotron Weiss Gallenkamp, UK) with an area of $1.68 \mathrm{~m}^{2}$ and growing height of $1.4 \mathrm{~m}$. Plants were grown under a photoperiod of $16 \mathrm{~h}$ light with a PPFD of $500 \mu \mathrm{mol} \mathrm{m} \mathrm{m}^{-2} \mathrm{~s}^{-1}$; lights were on at 00:00 and off at 16:00 each day (Fig. S1). Air temperature was $20 / 18{ }^{\circ} \mathrm{C}$ day/night and the relative humidity was maintained at $60 \%$. Plants were watered daily. Plants were grown in two controlled environment cabinets to have sufficient replication, 45 plants ( 15 pots) were placed in each cabinet and the 
plants in the two cabinets were planted and harvested in two consecutive days.

The experimental design was a split-plot in two blocks (i.e. two cabinets). Each sample was taken from a separate plant, and three samples were taken at each time point from each cabinet, resulting in six individual samples (i.e. six biological replicates) per time of the day, three from each of the two cabinets. The samples were taken throughout the diel cycle at 20:00, 22:00, 23:00, 24:00, 01:00, 02:00, 04:00, 06:00, 08:00, 10:00, 12:00, 14:00, 16:00, 17:00 and 18:00 h, with good representation of the period in darkness and in the light (Fig. S1).

The youngest fully expanded leaf with a visible collar, from the main tiller, was harvested 38 days after planting (growth stage Zadoks 2.5-3.0; Zadoks et al. 1974). The youngest fully expanded leaf was generally the fourth leaf, but in some more developed plants it corresponded to the fifth leaf. A section of $8 \mathrm{~cm}$ in the middle of the leaf, avoiding the leaf base and tip, was divided into four 2-cm-long segments and two of each of these used for each of two subsamples: one for quantifying Rca and Rubisco protein abundance and another for gene expression analysis by qRT-PCR.

\section{Gene expression and quantification}

Gene expression of Rca (TaRca1- $\beta$, TaRca2- $\beta$, TaRca2- $\alpha$ ), and Rubisco large and small subunits (TarbcL and $T a R b c S$ ), were determined by Real-Time Quantitative Reverse Transcription PCR (RT-qPCR). The extraction of total RNA from the wheat $\sim 4 \mathrm{~cm}^{2}$ leaf samples was completed using a modified hot phenol method (Shinmachi et al. 2010; Verwoerd et al. 1989). The total RNA concentration and quality was determined by measuring the absorbance at 230, 260 and $280 \mathrm{~nm}$ with a Nanodrop spectrometer (Thermo Fisher Scientific, Inc., UK) and running on a $1 \%(\mathrm{w} / \mathrm{v})$ agarose gel. A sub-sample of $1 \mu \mathrm{g}$ of total RNA was used for cDNA synthesis, using Superscript III as per the manufacturer's instructions (Life Technologies Ltd., UK).

For qRT-PCR a 1:10 dilution of cDNA was used with SYBRGreen (Platinum ${ }^{\circledR}$ SYBR ${ }^{\circledR}$ Green qPCR SuperMixUDG w/ROX, Life Technologies, UK), in $25 \mu \mathrm{L}$ reactions, as per the manufacturer's instructions. Primer pairs specific to TaRca1- $\beta$, TaRca2- $\beta$, TaRca2- $\alpha$, TarbcL and TaRbcS (primers were specific for TaRbcS located on chromosome 5A, 5B and 5D, group S3 as described by Degen et al. 2021), were used for qRT-PCR, alongside primers for two reference genes (Table S1). Primers were designed to bind to all three wheat sub-genomes, except for $r b c L$, which is encoded in the chloroplast genome. The qRT-PCR conditions were: $50{ }^{\circ} \mathrm{C}$ for $2 \mathrm{~min}, 95^{\circ} \mathrm{C}$ for $10 \mathrm{~min}$, followed by 40 cycles of $95^{\circ} \mathrm{C}$ for $15 \mathrm{~s}$ and $60{ }^{\circ} \mathrm{C}$ for $1 \mathrm{~min}$. Melt curves were also completed: $95{ }^{\circ} \mathrm{C}$ for $15 \mathrm{~s}, 60{ }^{\circ} \mathrm{C}$ for $1 \mathrm{~min}$ and $95{ }^{\circ} \mathrm{C}$ for
15 s (7500 Real-Time PCR machine, Applied Biosystems, Life Technologies, UK).

The mean primer efficiency was estimated using the linear phase of all individual reaction amplification curves (Ramakers et al. 2003) and calculated using the LinRegPCR package (Tuomi et al. 2010). The actin and succinate dehydrogenase genes were used as reference genes for the normalised relative quantification of expression. The normalised relative quantity (NRQ) of expression was calculated in relation to the cycle threshold (CT) values and the primer efficiency $(E)$ of the target gene $(X)$ and the normalising reference gene $(N)$, based on Rieu and Powers (2009): $\mathrm{NRQ}=(E X)^{-\mathrm{CT}, X} /(E N)^{-\mathrm{CT}, N}$.

\section{Rubisco and Rubisco activase protein quantification}

To determine the amounts of Rubisco and Rca protein in the wheat $\sim 4 \mathrm{~cm}^{2}$ leaf samples, extracts were prepared as described by Perdomo et al. (2018). Total soluble protein (TSP) concentration in the crude extracts was determined according to the Bradford (1976) method using bovine serum albumin as standard. Based on the TSP concentration all samples were diluted to $0.6 \mu \mathrm{g} \mu \mathrm{L}^{-1}$ and $3 \mu \mathrm{g}$ of TSP was used per sample for Rubisco and Rca quantification. Proteins were separated by sodium dodecyl sulphate polyacrylamide gel electrophoresis (SDS-PAGE) on 12\% gels hand casted and either visualised by staining with Coomassie Blue for Rubisco, or subject to immunoblotting for Rca. For the latter, proteins were transferred from the gel to a nitrocellulose membrane (iBlot, Thermofisher, UK), probed with a primary antibody anti-Rca produced in rabbit against cotton (Salvucci 2008) and a fluorescent secondary antibody for visualisation of Rca using an Odyssey Fc imaging (LI-COR, Lincoln, USA).

For Rca quantification, purified wheat Rca was used to load in each gel a series of four standards with increasing quantities, $0.01,0.05,0.1$ and $0.15 \mu \mathrm{g}$, to prepare a calibration curve (Fig. S2). For Rubisco quantification, purified wheat Rubisco was used to load in each gel a series of four standards with increasing quantities, $0.1,0.5,1.0$ and $1.5 \mu \mathrm{g}$, to prepare a calibration curve (Fig. S2).

\section{Data analysis and modelling}

Data were analysed using R 3.6.2 (R Core Team 2020) and RSTUDIO 1.2.5033 (RStudio Team 2020), and graphs were prepared using the GGPLOT 2 package (Wickham 2017). Linear Mixed Effects Regression (LMER) was used to assess the significance of differences in the gene expression and protein abundance of Rca and Rubisco between the different sampling times. Mean values and standard error of the mean (SEM) are shown in figures. To estimate maximum gene expression and protein amounts for Rca and Rubisco, and 
the corresponding times at which the maximum values were reached, second- to fourth-order polynomials and generalised additive models (GAM) were fitted to the experimental data using the gam function from the MGCV 1.8-24 package in R (Wood 2017). The model that best-fit the experimental data was selected based on the Akaike information criterion (Akaike 1974) using the AIC function (Table S2). The 'predict' function was used to estimate the maximum gene expression and protein amount values and the corresponding times for each Rca isoform and Rubisco subunits. The standard error calculated for each model was used to predict $95 \%$ confidence intervals for each fit (dashed lines in the graphs).

\section{Results}

\section{Gene expression of TaRca2 and TaRbcS peaked at 8-10 $\mathrm{h}$ into the photoperiod}

The expression of TaRcal and TaRca2 encoding the three Rca isoforms present in wheat (TaRcal- $\beta$, TaRca2- $\beta$ and TaRca2- $\alpha$ ), as well as the Rubisco large ( $r b c L)$ and small $(R b c S)$ subunits, were determined throughout the diel cycle using quantitative real-time PCR analysis. TaRca2- $\beta$ and TaRca2- $\alpha$ transcripts were much more abundant than TaRcal- $\beta$, which was detectable in very small amounts.
The expression of TaRca2- $\beta$ was highest at the middle of the light phase, $8 \mathrm{~h}$ into the photoperiod, while TaRca2- $\alpha$ had the highest expression levels occurring $10 \mathrm{~h}$ into the photoperiod. The expression of both TaRca2- $\beta$ and TaRca2- $\alpha$ decreased significantly in the second half of the light phase, reaching a minimum at the end of the light phase and remaining low and constant during the dark phase (Fig. 1). The ratio between the TaRca2- $\alpha$ and TaRca2- $\beta$ relative gene expression remained mostly constant throughout the diel cycle, with the expression of TaRca2 $-\alpha$ corresponding to approximately $85 \pm 3 \%$ relative to the TaRca2- $\beta$ isoform (Fig. S3).

The relative expression of the TaRcal gene was extremely low, with Normalised Relative Quantity values (NRQ) from 0.001 to 0.008 , which are much lower than its homologues encoded by the TaRca 2 gene. No significant differences in TaRcal- $\beta$ expression were detected between time points throughout the diel cycle.

The relative gene expression of the Rubisco subunits, TarbcL and TaRbcS, also fluctuated during the diel cycle; however, these fluctuations were only significant for the small subunit, $T a R b c S$, which showed lowest expression at $4 \mathrm{~h}$ into the photoperiod and highest expression at $10 \mathrm{~h}$ into the photoperiod (Fig. 2). The relative expression levels of both Rubisco subunits, TarbcL and TaRbcS, did not show a consistent trend during the dark phase, remaining mostly stable.

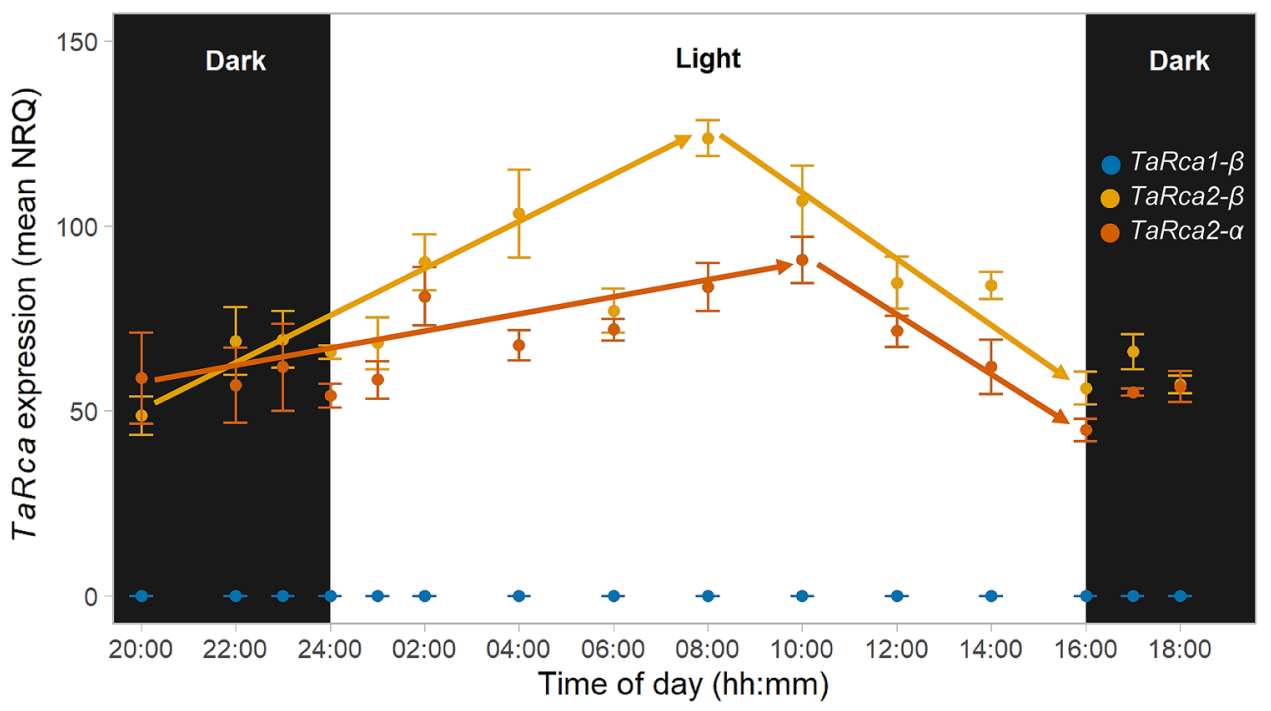

Fig. 1 TaRcal- $\beta$, TaRca2- $\beta$ and TaRca2- $\alpha$ expression throughout the diel cycle in young fully expanded leaves of wheat. Samples were taken from individual plants 38 days after planting (vegetative stage) at the indicated times during the night ("Dark", black background) and day ("Light", white background). Gene expression was estimated as normalised relative quantification (NRQ) using actin and succinate dehydrogenase as reference genes. Values are means \pm SEM $(n=4-6$ biological replicates). Arrows mean significant, positive and negative, correlations between gene expression and time of the day for TaRca $2 \beta$ and TaRca $2 \alpha$ (Pearson correlation analysis, $P<0.01$ ) 


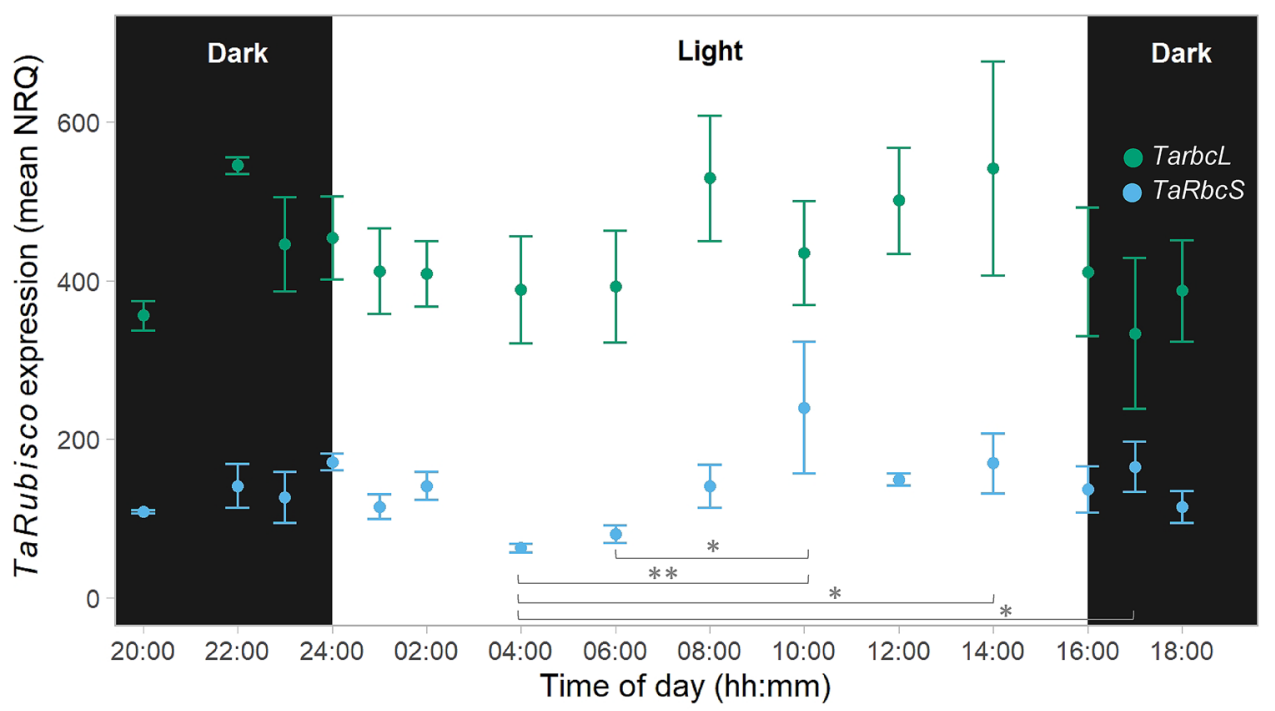

Fig. 2 TarbcL and TaRbcS expression throughout the diel cycle in young fully expanded leaves of wheat. Samples were taken from individual plants 38 days after planting (vegetative stage) at the indicated times during the night ("Dark", black background) and day ("Light", white background). Gene expression was estimated as normalised relative quantification (NRQ) using actin and succinate dehydrogenase as reference genes. $T a R b c S$ expression used primers specific to the

\section{TaRca- $\beta$ was more abundant than TaRca- $\alpha$ and the isoform ratio remained unchanged during the diel cycle}

The protein abundance of TaRca- $\beta$ and TaRca- $\alpha$ in young fully expanded leaves of wheat plants throughout the diel cycle was estimated by immunoblotting. Because of the barely detectable transcript abundance of TaRca1- $\beta$ (Fig. 1), we assumed that the antibody reactivity to the genes in chromosome 5 (group S3). Values are means \pm SEM $(n=4-6$ biological replicates). There was a significant effect of time of day on the expression of TaRbcS (ANOVA, $P<0.05$ ). The times of day which are significantly different are marked with asterisks [Tukey's honestly significant difference (HSD) mean-separation test, $* P<0.05$ and $* * P<0.01]$

shorter Rca- $\beta$ represents mainly TaRca $2-\beta$. TaRca- $\beta$ was much more abundant than TaRca- $\alpha$ during the entire diel cycle (Fig. 3). Although the TaRca- $\beta$ amounts appeared highest at $10 \mathrm{~h}$ into the photoperiod, there were no significant differences between the time points at which measurements were taken during the diel cycle. Similarly, TaRca- $\alpha$ remained constant during the entire diel cycle (Fig. 3). The protein ratio between the isoforms TaRca- $\alpha$ and TaRca- $\beta$ was $12.5 \pm 0.5 \%$ throughout the diel cycle (Fig. S3).
Fig. 3 TaRca- $\beta$ and TaRca- $\alpha$ protein amounts throughout the diel cycle in young fully expanded leaves of wheat. Samples were taken from individual plants 38 days after planting (vegetative stage) at the indicated times during the night ("Dark", black background) and day ("Light", white background). Rca amount was estimated by reference to a calibration curve prepared with increasing amounts of purified TaRca. Values are means \pm SEM ( $n=4-6$ biological replicates).

There were no significant differences between time of day and TaRca amount (ANOVA, $P>0.05$ )

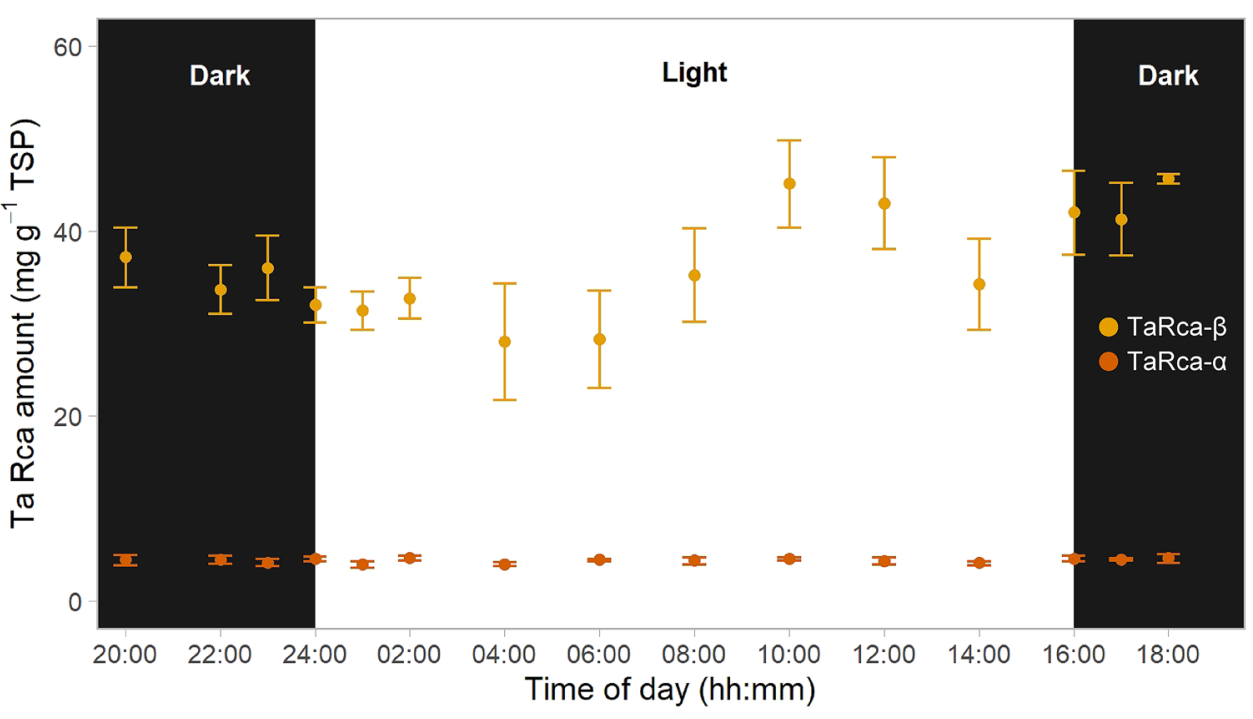




\section{Rubisco abundance decreased during the dark period}

The amount of Rubisco protein estimated by SDS-PAGE based on the abundance of the large subunit relative to total soluble protein remained constant throughout the photoperiod but decreased in the dark period (Fig. 4). Rubisco abundance was lowest $4 \mathrm{~h}$ into the dark period, with a significant difference between the value at the middle of the dark period $(20: 00 \mathrm{~h})$ and most of the values during the photoperiod. Moreover, there was a significant decrease in Rubisco abundance in the first half of the dark period followed by a significant increase in the second half of the photoperiod (Fig. 4). The higher level of abundance observed at the start of the photoperiod was maintained during the light phase.

\section{TaRca- $\beta$ exhibited a time-lag between gene expression and protein amount}

To estimate the maximum gene expression and protein amount for Rca isoforms and Rubisco, and the corresponding times at which the maximum values are obtained, a generalised additive model (GAM) was fitted to the experimental data (Figs. S4, S5). Based on the model predictions, TaRca2- $\beta$ and TaRca2- $\alpha$ had their maximum relative gene expression values (117.1 and 85.1 NRQ, respectively) at 09:15 h into the photoperiod (Table 1). By comparison, the maximum predicted amount of TaRca- $\beta$ protein was
Table 1 Predicted values for Rca and Rubisco transcript and protein abundance in wheat

\begin{tabular}{|c|c|c|c|}
\hline Isoform/subunit & Predicted value & $\begin{array}{l}\text { Time } \\
\text { of day } \\
\text { (hh:mm) }\end{array}$ & $P$ value \\
\hline TaRcal- $\beta$ (mean NRQ) & 0.002 (Mean) & NA & 0.583 \\
\hline TaRca2- $\beta$ (mean NRQ) & 117.1 (Max) & $09: 15$ & $<0.001 * * *$ \\
\hline TaRca2- $\alpha$ (mean NRQ) & 85.1 (Max) & $09: 15$ & $<0.001 * * *$ \\
\hline TaRca- $\beta$ (mg g ${ }^{-1}$ TSP) & 42.5 (Max) & $10: 45$ & $<0.01 * *$ \\
\hline TaRca- $\alpha\left(\mathrm{mg} \mathrm{g}^{-1}\right.$ TSP $)$ & 4.35 (Mean) & NA & 0.411 \\
\hline TarbcL (mean NRQ) & 446.1 (Mean) & NA & 0.594 \\
\hline$T a R b c S$ (mean NRQ) & 185.6 (Max) & $10: 30$ & $<0.05^{*}$ \\
\hline TarbcL (mg g ${ }^{-1}$ TSP) & 770.0 (Max) & 01:00 & $<0.001 * * *$ \\
\hline
\end{tabular}

The maximum and mean abundances, the times at which these occurred and the respective $P$ values $(* P<0.05$, $* * P<0.01$ and $* * * P<0.001)$ were estimated from the best-fit models applied to describe the diurnal response of Rca and Rubisco gene expression and protein amounts (Figs. S4, S5; Table S2)

$42.5 \mathrm{mg} \mathrm{g}^{-1}$ TSP at 10:45 $\mathrm{h}(1.5 \mathrm{~h}$ after the peak in gene expression), and there was no peak for TaRca- $\alpha$ protein during the photoperiod.

Conversely, for Rubisco, there was a predicted peak in the large subunit protein amount at 01:00 $\mathrm{h}$ into the photoperiod, with no corresponding peak in gene expression, which remained constant throughout the light period (Table 1). These results show a lag between gene expression and

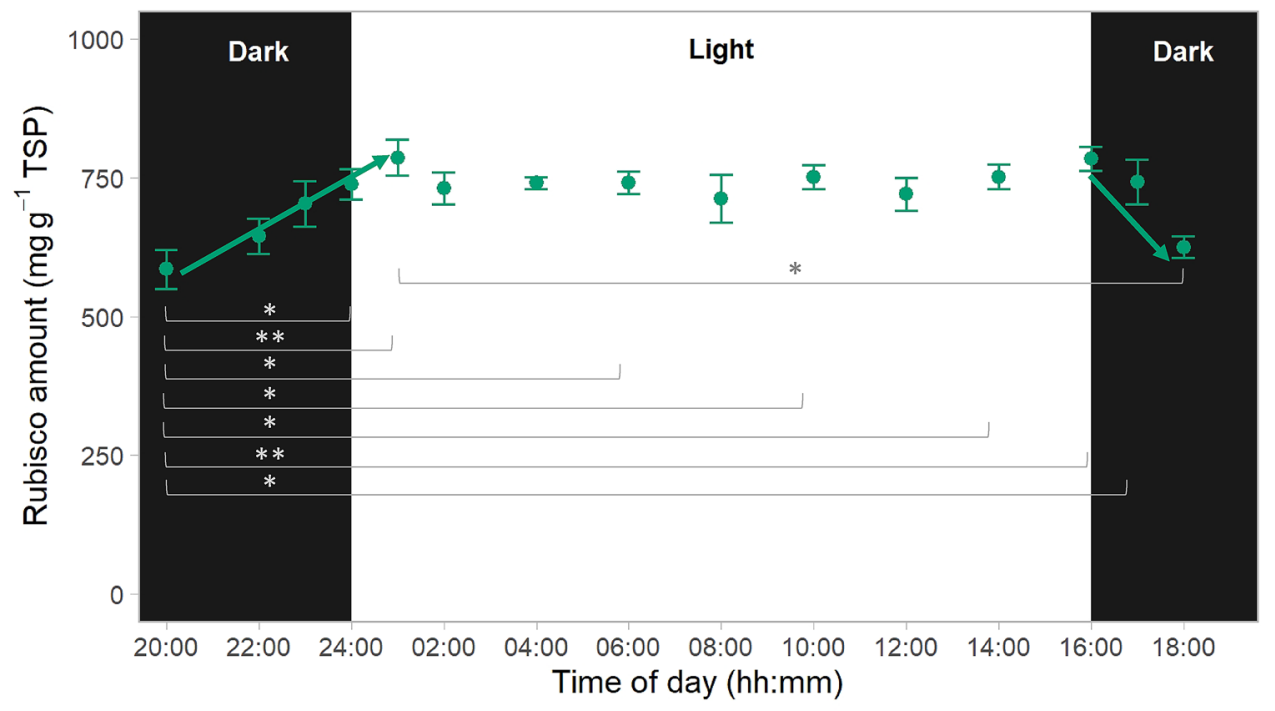

Fig. 4 Rubisco amount throughout the diel cycle in young fully expanded leaves of wheat. Samples were taken from individual plants 38 days after planting (vegetative stage) at the indicated times during the night ("Dark", black background) and day ("Light", white background). Rubisco amount was estimated by reference to a calibration curve prepared with increasing amounts of purified enzyme and based on density of the large subunit (TarbcL). Values are means \pm SEM ( $n=4-6$ biological replicates). The times of day which are significantly different are marked with asterisks [Tukey's honestly significant difference (HSD) mean-separation test, $* P<0.05$ and $* * P<0.01]$. Arrows mean significant, positive and negative, correlations between Rubisco amount and time of the day (Pearson correlation analysis, $P<0.05$ ) 
protein abundance for TaRca- $\beta$, and a generalised lack of correlations between gene expression and protein abundance of Rubisco and Rca during the diel cycle (Fig. S6).

\section{Discussion}

Rubisco activase (Rca), a molecular chaperone required to maintain Rubisco activity, occurs in diverse isoforms. In wheat, three isoforms have been described that are known to differ in their redox-sensitivity (by inference from results with Arabidopsis; Zhang and Portis, 1999; Zhang et al. 2001) and inhibition by ADP (Perdomo et al. 2019; Degen et al. 2020). Here, we show that the relative abundance of the redox-sensitive TaRca- $\alpha$ and redox-insensitive TaRca- $\beta$ isoforms remains constant in the leaves of wheat plants grown under controlled conditions throughout the entire diel cycle. TaRca- $\alpha$ isoform is less abundant, representing $85 \%$ of the redox-insensitive TaRca- $\beta$ at the transcript level and $12.5 \%$ at the protein level.

$R c a$ gene expression has been reported to respond to organ-specific signals, light availability and the circadian clock in a species-specific manner (Orozco and Ogren 1993; Watillon et al. 1993; Liu et al. 1996; Chao et al. 2014). The expression of the wheat Rca isoforms TaRca2- $\alpha$ and TaRca2- $\beta$, encoded by the highly expressed TaRca 2 gene, increased during the first half of the photoperiod, with a peak at $8-10 \mathrm{~h}$ after the start of the $16 \mathrm{~h}$ photoperiod. The expression of TaRcal- $\beta$ isoform was extremely low, in agreement with previous reports (Scafaro et al. 2019a; Degen et al. 2021), and remained low throughout the entire diel cycle (Fig. 1). Rca expression peaked earlier in the day for other species previously characterised: $3 \mathrm{~h}$ after the start of a $14 \mathrm{~h}$ photoperiod in rice (To et al. 1999), $6 \mathrm{~h}$ after the start of a $14 \mathrm{~h}$ photoperiod in Arabidopsis (Pilgrim and McClung 1993), $2 \mathrm{~h}$ after the start of a $14 \mathrm{~h}$ photoperiod in soybean (Chao et al. 2014), and $2 \mathrm{~h}$ after the start of a $16 \mathrm{~h}$ photoperiod in apple (Watillon et al. 1993). It is also possible that the relative expression of Rca isoforms in different species may vary with growth conditions, plant age and developmental stage.

The expression of Rubisco large $(r b c L)$ and small $(R b c S)$ subunits is controlled by different internal and external factors such as light signals and temperature (Rodermel et al. 1996; Spreitzer 2003; Recuenco-Muñoz et al. 2015). The expression of TarbcL remained constant during the diel cycle, while $T a R b c S$ transcripts abundance was highest $10 \mathrm{~h}$ into the photoperiod (Fig. 2). Of note is that the primers used were specific to the $T a R b c S$ genes in chromosome 5 (group S3). As described by Degen et al. (2021), TaRbcS genes can be grouped according to their sequence similarity, with gene groups $\mathrm{S} 1$ and $\mathrm{S} 2$ in chromosome 2 and group S3 in chromosome 5. In the same study, the abundance of TaRbcS2 and TaRbcS3 transcripts was comparable and higher than $T a R b c S 1$ in wheat leaves that were sampled only at $4 \mathrm{~h}$ after the start of a $16 \mathrm{~h}$ photoperiod. The results presented here are in agreement with previous studies reporting that the Rubisco $R b c S$ is regulated by the circadian rhythm, while the $r b c L$ is not (Pilgrim and McClung 1991; Cheng et al. 1998; Recuenco-Muñoz et al. 2015). In Arabidopsis the peak in expression of $R b c S$ was reported earlier in the light phase, i.e. $1 \mathrm{~h}$ after the start of a $14 \mathrm{~h}$ photoperiod, and in C. reinhardtii and tomato $R b c S$ expression remained largely constant throughout the diel cycle (Martino-Catt and Ort 1992; Pilgrim and McClung 1993; Cheng et al. 1998; RecuencoMuñoz et al. 2015), suggesting a species-specific pattern. Whether TaRbcS1 and TaRbcS2 show a peak of expression at the middle of the photoperiod as observed for TaRbcS3 remains to be determined.

Rubisco protein abundance was highest at the start of the photoperiod and remained constant during the light phase. The $\mathrm{CO}_{2}$-fixing enzyme represents a large fraction of the total soluble protein (TSP) in wheat leaves, both in plants grown under controlled conditions (Fig. 4) and in the field (Carmo-Silva et al. 2015, 2017). The decrease in Rubisco abundance observed during the night period in the present study suggests significant degradation of the enzyme in the first $4 \mathrm{~h}$, followed by synthesis prior to the start of the photoperiod. The extent of Rubisco degradation cannot be accurately determined by SDS-PAGE and requires further investigation using more robust methods, including CABPbinding (Ferreira et al. 2000; Whitney and Sharwood 2014). Previous work has suggested that Rubisco is degraded at a slow rate both in rice (Mae et al. 1983; Suzuki et al. 2001; Irving and Robinson 2006) and in Arabidopsis (Li et al. 2017; Tivendale et al. 2020). Esquível et al. (1998) found the rate of Rubisco degradation to be species-specific; and it is likely that the degradation rate changes with leaf and plant age, as well as with environmental conditions. Importantly, large daily fluctuations of the most abundant leaf protein represent a significant cellular energetic burden, and Rubisco abundance has been shown to impact wheat grain yields (e.g. Lobo et al. 2019). Thus, further investigation is warranted into the rates of Rubisco synthesis and degradation, and the regulation of Rubisco protein abundance and turnover.

Variation in Rubisco activity during the photoperiod is more likely to be controlled via regulation of its activity than protein abundance (Li et al. 2020; Davies and Griffiths 2012). The molecular chaperone Rca is a key player in the regulation of Rubisco activity. Modelling of Rca isoform protein amounts suggested a peak in the abundance of TaRca- $\beta$ at 10:45 h into the photoperiod (Table 1), while TaRca- $\alpha$ abundance remained unchanged throughout the diel cycle. In tomato, Rca abundance peaked $2 \mathrm{~h}$ before the start of the photoperiod and was lowest at the middle of the photoperiod (Martino-Catt and Ort 1992). The ratio between 
total Rca and Rubisco abundance remained largely constant during the photoperiod, suggesting sufficient amount of the molecular chaperone to maintain Rubisco activity through the release of inhibitory compounds from active sites.

The relative abundance of the different Rca isoforms differs among species. In Arabidopsis, Camelina and spinach, equal amounts of Rca- $\alpha$ and Rca- $\beta$ are present, while rice and soybean accumulate much more Rca- $\beta$ than Rca- $\alpha$ (Salvucci et al. 1987; Fukayama et al. 2012; Chao et al. 2014; Scafaro et al. 2016). Similarly, in wheat, TaRca- $\beta$ is much more abundant than TaRca- $\alpha$ (Law and Crafts-Brandner 2001; Degen et al. 2021), and the Rca- $\alpha / \beta$ ratio remained constant during the whole diel cycle $(12.5 \%$, Fig. S3). The three wheat Rca isoforms have been shown to differ in the sensitivity of Rubisco activation activity to inhibition by ADP (Perdomo et al. 2019; Scafaro et al. 2019b), as well as in response to temperature (Scafaro et al. 2019a; Degen et al. 2020). It would be conceivable that plants might up-regulate the abundance of one or another isoform in adaptation to the prevailing environment. Accordingly, the Rca- $\alpha / \beta$ ratio has been reported to increase in Brachypodium distachyon plants under drought and salinity stress (Bayramov and Guliyev 2014), and the relative abundance of TaRca1- $\beta$ increased in wheat at high temperature (Law and Crafts-Brandner 2001; Degen et al. 2021).

Coordination between gene expression and protein translation enables physiological responses to various environmental stimuli, essential for successful plant growth and reproduction (Grabsztunowicz et al. 2017). However, for both Rca isoforms and Rubisco, there was a mismatch between the time of day corresponding to the predicted maximum levels of transcript abundance and protein abundance (Table 1). While for TaRca- $\beta$ the maximum protein abundance was predicted to occur $1.5 \mathrm{~h}$ after the predicted peak of transcript abundance, for TaRca- $\alpha$ and for Rubisco there was a peak for transcript abundance and not for protein abundance or vice-versa. Moreover, the ratio TaRca- $\alpha /$ TaRca- $\beta$ was $85 \%$ at the transcript level and $12.5 \%$ at the protein level, and there was no significant correlation between gene expression and protein abundance for Rca and Rubisco during the diel cycle (Fig. S6). These findings agree with previous studies in wheat and other species under control and stress conditions, suggesting that Rca is regulated at either the translational level by mRNA silencing or at post-translational level by more rapid turnover of the protein (Law and Crafts-Brandner 2001; DeRidder et al. 2012; Bayramov and Guliyev 2014). A lack of correlation between gene expression and protein synthesis has also been reported for Rubisco in Arabidopsis, tobacco, rice and $C$. reinhardtii (Pilgrim and McClung 1993; Rodermel et al. 1996; Wang and Wang 2011; Recuenco-Muñoz 2015), again suggesting that abundance of the $\mathrm{CO}_{2}$-fixing enzyme might be regulated post-transcriptionally (Rodermel et al. 1996; Law and Crafts-Brandner 2001; Houtz and Portis 2003).

In summary, the results presented here show that the redox-sensitive TaRca- $\alpha$ isoform is less abundant than the redox-insensitive TaRca- $\beta$ isoform, the difference in abundance is more pronounce at the protein level $(12.5 \%$ $\alpha / \beta)$ than at the transcript level $(85 \% \alpha / \beta)$, and the ratio TaRca- $\alpha /$ TaRca- $\beta$ remains unchanged throughout the diel cycle. These results, combined with the lack of correlation between transcript and protein abundance for both Rca and Rubisco, suggest that the abundance of both enzymes and their isoforms is post-transcriptionally regulated.

Supplementary Information The online version contains supplementary material available at https://doi.org/10.1007/s11120-021-00830-6.

Acknowledgements We thank Dr. Mike Salvucci for the gift of Rca antibody, Dr. Stephen Powers for the contribution of the experimental design, Dr. Dawn Worrall for providing constructive feedback on the manuscript, Mr. Anthony Griffin for help in plant growth and maintenance and Miss Abbie Rogan for help with RNA extractions.

Author contributions E.C.S. designed the experiments and supervised the project. J.A.P. carried out the experiment and the laboratory assays. P.B. contributed to gene expression analysis. J.A.P. analysed the data. J.A.P. and E.C.S. wrote the manuscript with contributions from P.B.

Funding This research was funded by the Biotechnology and Biological Sciences Research Council (BBSRC) through the International Wheat Yield Partnership Project Speeding the adjustment of photosynthesis to shade-sun transitions to increase yield potential in the field (IWYP163; BB/S005072/1), and the Rothamsted Research Institute Strategic Programs 20:20 Wheat ${ }^{\circledR}$ (BBSRC BB/J/00426X/1 20:20 Wheat ${ }^{\circledR}$ ) and Designing Future Wheat (BBSRC BB/P016855/1). J.A.P. was initially supported through a Rothamsted Research Fellowship awarded to E.C.S.

\section{Declarations}

Conflict of interest The Authors declare that there are no competing interests associated with the manuscript.

Open Access This article is licensed under a Creative Commons Attribution 4.0 International License, which permits use, sharing, adaptation, distribution and reproduction in any medium or format, as long as you give appropriate credit to the original author(s) and the source, provide a link to the Creative Commons licence, and indicate if changes were made. The images or other third party material in this article are included in the article's Creative Commons licence, unless indicated otherwise in a credit line to the material. If material is not included in the article's Creative Commons licence and your intended use is not permitted by statutory regulation or exceeds the permitted use, you will need to obtain permission directly from the copyright holder. To view a copy of this licence, visit http://creativecommons.org/licenses/by/4.0/. 


\section{References}

Akaike H (1974) A new look at the statistical model identification. IEEE Trans Autom Control 19:716-723

Bayramov S, Guliyev N (2014) Changes in Rubisco activase gene expression and polypeptide content in Brachypodium distachyon. Plant Physiol Biochem 81:61-66

Berry JA, Downton WJS (1982) Environmental regulation of photosynthesis. Photosynthesis 2:263-343

Bradford MM (1976) A rapid and sensitive method for the quantitation of microgram quantities of protein utilizing the principle of protein-dye binding. Anal Biochem 72:248-254

Brooks A, Portis AR Jr (1988) Protein-bound ribulose bisphosphate correlates with deactivation of ribulose bisphosphate carboxylase in leaves. Plant Physiol 87:244-249

Carmo-Silva E, Scales JC, Madgwick PJ, Parry MAJ (2015) Optimizing Rubisco and its regulation for greater resource use efficiency. Plant Cell Environ 38:1817-1832

Carmo-Silva E, John Andralojc P, Scales JC, Driever SM, Mead A, Lawson T, Raines CA, Parry MAJ (2017) Phenotyping of fieldgrown wheat in the UK highlights contribution of light response of photosynthesis and flag leaf longevity to grain yield. J Exp Bot 68(13):3473-3486

Chao M, Yin Z, Hao D, Zhang J, Song H, Ning A et al (2014) Variation in Rubisco activase (RCA $\beta$ ) gene promoters and expression in soybean [Glycine $\max (\mathrm{L}$.$) Merr.]. J Exp Bot 65:47-59$

Cheng S, Moore B, Seemann JR (1998) Effects of short- and longterm elevated $\mathrm{CO}_{2}$ on the expression of ribulose-1,5-bisphosphate carboxylase/oxygenase genes and carbohydrate accumulation in leaves of Arabidopsis thaliana (L.) Heynh. Plant Physiol 116:715-723

Cohen I, Sapir Y, Shapira M (2006) A conserved mechanism controls translation of Rubisco large subunit in different photosynthetic organisms. Plant Physiol 141:1089-1097

Davies BN, Griffiths H (2012) Competing carboxylases: Circadian and metabolic regulation of Rubisco in $\mathrm{C}_{3}$ and CAM Mesembryanthemum crystallinum L. Plant Cell Environ 35:1211-1220

Degen GE, Worrall D, Carmo-Silva E (2020) An isoleucine residue acts as a thermal and regulatory switch in wheat Rubisco activase. Plant J 103:742-751

Degen GE, Orr DJ, Carmo-Silva E (2021) Heat-induced changes in the abundance of wheat Rubisco activase isoforms. N Phytol 229:1298-1311

DeRidder BP, Shybut ME, Dyle MC, Kremling KAG, Shapiro MB (2012) Changes at the 3'-untranslated region stabilize Rubisco activase transcript levels during heat stress in Arabidopsis. Planta 236:463-476

Esquível MG, Ferreira RB, Teixeira AR (1998) Protein degradation in $\mathrm{C}_{3}$ and $\mathrm{C}_{4}$ plants with particular reference to ribulose bisphosphate carboxylase and glycolate oxidase. J Exp Bot 49:807-816

Ferreira RB, Esquível MG, Teixeira AR (2000) An accurate method to quantify ribulose bisphosphate carboxylase content in plant tissue. Plant Cell Environ 23:1329-1340

Fukayama H, Ueguchi C, Nishikawa K, Katoh N, Ishikawa C, Masumoto C, Hatanaka T, Misoo S (2012) Overexpression of Rubisco activase decreases the photosynthetic $\mathrm{CO}_{2}$ assimilation rate by reducing Rubisco content in rice leaves. Plant Cell Physiol 53 (6):976-986

Grabsztunowicz M, Koskela MM, Mulo P (2017) Post-translational modifications in regulation of chloroplast function: recent advances. Front Plant Sci 8:1-12

Harmer SL (2009) The circadian system in higher plants. Annu Rev Plant Biol 60:357-377
Houtz RL, Portis AR Jr (2003) The life of ribulose 1,5-bisphosphate carboxylase/oxygenase-posttranslational facts and mysteries. Arch Biochem Biophys 414:150-158

Irving LJ, Robinson D (2006) A dynamic model of Rubisco turnover in cereal leaves. N Phytol 169:493-504

Jordan DB, Chollet R (1983) Inhibition of ribulose bisphosphate carboxylase by substrate ribulose. J Biol Chem 258:13752-13758

Klein RR, Salvucci ME (1995) Rubisco, rubisco activase and ribulose5-phosphate kinase gene expression and polypeptide accumulation in a tobacco mutant defective in chloroplast protein synthesis. Photosynth Res 43:213-223

Law RD, Crafts-Brandner SJ (2001) High temperature stress increases the expression of wheat leaf ribulose-1,5-bisphosphate carboxylase/oxygenase activase protein. Arch Biochem Biophys 386:261-267

Li J, Yokosho K, Liu S, Cao HR, Yamaji N, Zhu XG et al (2020) Diel magnesium fluctuations in chloroplasts contribute to photosynthesis in rice. Nat Plants 6:848-859

Li L, Nelson CJ, Trösch J, Castleden I, Huang S, Millar AH (2017) Protein degradation rate in Arabidopsis thaliana leaf growth and development. Plant Cell 29:207-228

Liu Y, Beyer A, Aebersold R (2016) On the dependency of cellular protein levels on mRNA abundance. Cell 165:535-550

Liu Z, Taub CC, McClung CR (1996) Identification of an Arabidopsis thaliana ribulose-1,5-bisphosphate carboxylase oxygenase activase (RCA) minimal promoter regulated by light and the circadian clock. Plant Physiol 112:43-51

Lobo AKM, Orr DJ, Gutierrez MO, Andralojc PJ, Sparks C, Parry MAJ, Carmo-Silva E (2019) Overexpression of calpase decreases Rubisco abundance and grain yield in wheat. Plant Physiol 181:471-479

Mae T, Makino A, Ohira K (1983) Changes in the amounts of ribulose bisphosphate carboxylase synthesized and degraded during the life span of rice leaf (Oryza sativa L.). Plant Cell Physiol 24:1079-1086

Martino-Catt S, Ort DR (1992) Low temperature interrupts circadian regulation of transcriptional activity in chilling-sensitive plants. Proc Natl Acad Sci USA 89:3731-3735

Neuwald AF, Aravind L, Spouge JL, Koonin EV (1999) AAA+: a class of chaperone-like ATPases associated with the assembly, operation, and disassembly of protein complexes. Genome Res 9:27-43

Orozco BM, Ogren WL (1993) Localization of light-inducible and tissue-specific regions of the spinach ribulose bisphosphate carboxylase/oxygenase (Rubisco) activase promoter in transgenic tobacco plants. Plant Mol Biol 23:1129-1138

Perdomo JA, Degen GE, Worrall D, Carmo-Silva E (2019) Rubisco activation by wheat Rubisco activase isoform $2 \beta$ is insensitive to inhibition by ADP. Biochem J 476:2595-2606

Perdomo JA, Sales CRG, Carmo-Silva E (2018) Quantification of photosynthetic enzymes in leaf extracts by immunoblotting. In: Covshoff S (ed) Photosynthesis. Methods in molecular biology. Humana Press, New York, pp 215-227

Pilgrim ML, McClung CR (1993) Differential involvement of the circadian clock in the expression of genes required for ribulose1,5-bisphosphate carboxylase/oxygenase synthesis, assembly, and activation in Arabidopsis thaliana. Plant Physiol 103:553-564

Portis AR Jr (1995) The regulation of Rubisco by Rubisco activase. J Exp Bot 46:1285-1291

R Core Team (2020) R: a language and environment for statistical computing. R Foundation for Statistical Computing, Vienna. https:// www.R-project.org/

Ramakers C, Ruijter, Lekanne Deprez RH, Moorman AFM (2003) Assumption-free analysis of quantitative real-time polymerase chain reaction (PCR) data. Neurosci Lett 339(1):62-66 
Ray DK, Mueller ND, West PC, Foley JA (2013) Yield trends are insufficient to double global crop production by 2050 . PLoS ONE $8: 1-8$

Recuenco-Muñoz L, Offre P, Valledor L, Lyon D, Weckwerth W, Wienkoop S (2015) Targeted quantitative analysis of a diurnal RuBisCO subunit expression and translation profile in Chlamydomonas reinhardtii introducing a novel Mass Western approach. J Proteomics 113:143-153

Rieu I, Powers SJ (2009) Real-time quantitative RT-PCR: design, calculations, and statistics. Plant Cell 21:1031-1033

Rodermel S, Haley J, Jiang CZ, Tsai CH, Bogorad L (1996) A mechanism for intergenomic integration: abundance of ribulose bisphosphate carboxylase small-subunit protein influences the translation of the large-subunit mRNA. Proc Natl Acad Sci USA 93:3881-3885

Roy H (1989) Rubisco assembly: a model system for studying the mechanism of chaperonin action. Plant Cell 1:1035-1042

RStudio Team (2020) RStudio: integrated development for R. RStudio, PBC, Boston. http://www.rstudio.com/

Salvucci ME, Werneke J, Ogren W, Portis AR Jr (1987) Purification and species distribution of Rubisco activase. Plant Physiol 84:930-936

Salvucci ME, Van de Loo FJ, Stecher D (2003) Two isoforms of Rubisco activase in cotton, the products of separate genes not alternative splicing. Planta 216:736-744

Salvucci ME (2008) Association of Rubisco activase with chaperonin60 $\beta$ : a possible mechanism for protecting photosynthesis during heat stress. J Exp Bot 59:1923-1933

Scafaro AP, Bautsoens N, den Boer B, Van Rie J, Gallé A (2019a) A conserved sequence from heat-adapted species improves Rubisco activase thermostability in wheat. Plant Physiol 181:43-54

Scafaro AP, De Vleesschauwer D, Bautsoens N, Hannah MA, den Boer B, Gallé A, Van Rie J (2019b) A single point mutation in the C-terminal extension of wheat Rubisco activase dramatically reduces ADP inhibition via enhanced ATP binding affinity. J Biol Chem 294:17931-17940

Scafaro AP, Gallé A, Van Rie J, Carmo-Silva E, Salvucci ME, Atwell BJ (2016) Heat tolerance in a wild Oryza species is attributed to maintenance of Rubisco activation by a thermally stable Rubisco activase ortholog. N Phytol 211:899-911

Schmidt GW, Mishkind ML (1986) The transport of proteins into chloroplasts. Annu Rev Biochem 55:879-912

Shinmachi F, Buchner P, Stroud JL, Parmar S, Zhao FJ, Mcgrath SP, Hawkesford MJ (2010) Influence of sulfur deficiency on the expression of specific sulfate transporters and the distribution of sulfur, selenium, and molybdenum in wheat. Plant Physiol 153:327-336

Spreitzer RJ (2003) Role of the small subunit in ribulose-1,5-bisphosphate carboxylase/oxygenase. Arch Biochem Biophys 414:141-149

Suzuki Y, Makino A, Mae T (2001) Changes in the turnover of Rubisco and levels of mRNAs of $r b c L$ and $r b c S$ in rice leaves from emergence to senescence. Plant Cell Environ 24:1353-1360

Tivendale ND, Hanson AD, Henry CS, Hegeman AD, Millar AH (2020) Enzymes as parts in need of replacement-and how to extend their working life. Trends Plant Sci 25:7

To K, Suen D, Chen SG (1999) Molecular characterization of ribulose1,5-bisphosphate carboxylase/oxygenase activase in rice leaves. Planta 209:66-76
Tuomi JM, Voorbraak F, Jones DL, Ruijter JM (2010) Bias in the Cq value observed with hydrolysis probe based quantitative PCR can be corrected with the estimated PCR efficiency value. Methods 50:313-322

Verwoerd TC, Dekker BMM, Hoekema A (1989) A small-scale procedure for the rapid isolation of plant RNAs. Nucleic Acids Res 17:2362

Vogel C, Marcotte EM (2012) Insights into the regulation of protein abundance from proteomic and transcriptomic analyses. Nat Rev Genet 13:227-232

Wang Z, Wang T (2011) Dynamic proteomic analysis reveals diurnal homeostasis of key pathways in rice leaves. Proteomics 11:225-238

Watillon B, Kettmann R, Boxus P, Burny A (1993) Developmental and circadian pattern of Rubisco activase mRNA accumulation in apple plants. Plant Mol Biol 23:501-509

Werneke JM, Chatfield JM, Ogren WL (1989) Alternative mRNA splicing generates the two ribulose bisphosphate carboxylase/ oxygenase activase polypeptides in spinach and Arabidopsis. Plant Cell 1:815-825

Whitney SM, Sharwood RE (2014) Plastid transformation for Rubisco engineering and protocols for assessing expression. In: Maliga $\mathrm{P}$ (ed) Chloroplast biotechnology. Methods in molecular biology (methods and protocols). Humana Press, Totowa, pp 245-262

Wickham H. (2017) tidyverse: easily install and load "Tidyverse" packages. https://tidyverse.tidyverse.org/

Winder TL, Anderson JC, Spalding MH (1992) Translational regulation of the large and small subunits of ribulose bisphosphate carboxylase/oxygenase during induction of the $\mathrm{CO}_{2}$-concentrating mechanism in Chlamydomonas reinhardtii. Plant Physiol 98:1409-1414

Wood SN (2017) Generalized additive models: an introduction with R. CRC Press, Boca Raton

Yin Z, Meng F, Song H, Wang X, Xu X, Yu D (2010) Expression quantitative trait loci analysis of two genes encoding Rubisco activase in soybean. Plant Physiol 152:1625-1637

Zadoks JC, Chang TT, Konzak CF (1974) A decimal code for the growth stages of cereals. Weed Res 14:415-421

Zhang N, Portis AR Jr (1999) Mechanism of light regulation of Rubisco: a specific role for the larger Rubisco activase isoform involving reductive activation by thioredoxin-f. Proc Natl Acad Sci USA 96:9438-9443

Zhang N, Schürmann P, Portis AR Jr (2001) Characterization of the 579 regulatory function of the $46-\mathrm{kDa}$ isoform of Rubisco activase from 580 Arabidopsis. Photosynth Res 68:29-37

Zhao G, Xu H, Zhang P, Su X, Zhao H (2017) Effects of 2,4-epibrassinolide on photosynthesis and Rubisco activase gene expression in Triticum aestivum L. seedlings under a combination of drought and heat stress. Plant Growth Regul 81:377-384

Publisher's Note Springer Nature remains neutral with regard to jurisdictional claims in published maps and institutional affiliations. 